\title{
Onset of retinopathy of prematurity as related to postnatal and postconceptional age
}

\author{
Graham E Quinn, Lois Johnson, Soraya Abbasi
}

\begin{abstract}
The hypothesis that both perinatal events and stage of retinal development are important factors in determining the age at onset of retinopathy of prematurity (ROP) was tested by comparing gestational age at birth with postnatal and postconceptional age when ROP (using ICROP) was first seen. The study population consisted of 207 infants (111 placebo $(P)$ treated, 96 vitamin $E(E)$ treated) who developed ROP among a group of 914 premature infants $(460 \mathrm{P}, 454 \mathrm{E})$ enrolled in a randomised clinical trial of the effect of prophylactic use of vitamin $E$ at pharmacological serum levels on incidence and severity of retinopathy. The mean postnatal age at onset of retinopathy was delayed in $E$ treated infants compared with $P$ treated infants by 1.4 weeks $(t=4.004, p<0.0001)$. For both $P$ and $E$ treated infants postnatal age at onset of ROP (which reflects the state of retinal development at which birth insults occur) and postconceptional age at onset of ROP which defines state of maturity) were correlated with gestational age at birth. This suggests that both the event of premature birth and the extent of retinal development are important in determining when ROP will first be observed.
\end{abstract}

In this report the relationship of the postnatal age when retinopathy of prematurity (ROP) was first observed is compared with postconceptional age at first observation of ROP for infants born between 26 and 35 weeks of gestation. This observation provides insight into factors controlling initiation of abnormal vascularisation in the immature retina which characterises ROP. We also report the influence of the antioxidant vitamin $E(E)$ on the onset of ROP. The data that permit this analysis are derived from a group of 914 infants with birth weights (BW) of less than $2001 \mathrm{~g}$ or less than 37 weeks gestational age (GA) who were enrolled in a double blind, randomised clinical trial designed to evaluate the prophylactic use of $E$ at pharmacological serum levels $(5 \mathrm{mg}$ / $\mathrm{ml}$ ) in decreasing incidence and severity of ROP. Infants were treated prophylactically with either placebo $(\mathbf{P})$ or $\mathrm{E}$.

\section{Materials and methods}

\section{SUBJECTS AND STUDY MEDICATION} ADMINISTRATION

The study was undertaken at the Neonatal Complex of the University of Pennsylvania which includes the Infant Intensive Care Nurseries at Pennsylvania Hospital, The Hospital of the University of Pennsylvania and
The Children's Hospital of Philadelphia, the latter serving an entirely outborn population. Enrolment began on 1 January 1979 and ended 1 May 1981. Informed consent and enrolment were accomplished as soon as possible after birth but infants were eligible up to 5 days of age. Once enrolled infants were randomly assigned to a study number and the corresponding study medication (placebo or vitamin E) within five BW strata: $\leq 1000 \mathrm{~g}, 1001-1250 \mathrm{~g}, 1251-1500 \mathrm{~g}$, $1501-2000 \mathrm{~g}$, and $\geq 2000 \mathrm{~g}$ if $\leq 36$ weeks GA and requiring $>23$ hours of oxygen. Details regarding randomisation, method of study medication administration, laboratory analyses undertaken, the clinical data base recorded for each patient, and reasons for lack of follow-up are available in previous reports. ${ }^{12}$

\section{CLASSIFICATION AND SURVEILLANCE OF ROP}

ROP was characterised in this study using a classification system developed at the University of Pennsylvania ${ }^{34}$ prior to the cooperative efforts that led to the International Classification of ROP (ICROP). ${ }^{56}$ The former classification corresponds closely to ICROP. For the sake of clarity the conclusions presented in this report have been based on the widely used ICROP classification. The Schaffer-Quinn classification system defines vascular abnormalities characteristically seen in the earliest stage of ROP as also described by Patz ${ }^{6}$ and McCormick. ${ }^{7}$ These changes which are more difficult to define and standardise were not included in the ICROP classification. The demarcation line that characterises stage 1 ICROP corresponds to grade 2 in the Schaffer-Quinn classification. Stages 3,4 , and 5 are identical.

ROP surveillance began as soon as the neonatologist in charge felt that the infant could tolerate the procedure, usually in the first week of life and often in the first day or two of life Surveillance for retinopathy included weekly eye examinations which continued throughout the period of retinal vasculature immaturity or until any ROP observed had begun to regress or progressed to retinal detachment. Ophthalmic follow-up continued to age 3 years.

All examinations were carried out by one of

Table 1 Characteristics of study population

\begin{tabular}{lll}
\hline & $\begin{array}{l}\text { Birth weight } \\
(\mathrm{g})(S D)\end{array}$ & $\begin{array}{l}\text { Gestational age } \\
(\text { weeks })(S D)\end{array}$ \\
\hline $\begin{array}{l}\text { All placebo enrollees } \\
(n=460)\end{array}$ & $1444 \mathrm{~g}(439)$ & $31 \cdot 6(2 \cdot 8)$ \\
$\begin{array}{l}\text { All vit E enrollees } \\
(n=454)\end{array}$ & $1461 \mathrm{~g}(457)$ & $31 \cdot 5(2 \cdot 7)$ \\
$\begin{array}{l}\text { Placebo infants with ROP } \\
(n=111)\end{array}$ & $1149 \mathrm{~g}(295)$ & $29 \cdot 8(2 \cdot 3)$ \\
$\begin{array}{l}\text { Vit E infants with ROP } \\
(n=96)\end{array}$ & $1092 \mathrm{~g}(212)$ & $29 \cdot 4(2 \cdot 0)$ \\
\hline
\end{tabular}

西

?.

응

8을

$$
\text { ᄋ }
$$

글

Ophthalmology,
Children's Hospital of
Philadelphia and
Department of
Ophthalmology,
University of
Pennsylvania
G E Quinn
Correspondence to:
Graham E Quinn, MD,
Pediatric Ophthalmology,
Children's Hospital of
Philadelphia, One Children's
Center, Philadelphia, PA
19104, USA.
Accepted for publication
17 October 1991
17 October 1991
Division of Newborn
Pediatrics, Pennsylvan Hospital and Departmen of Pennsylvania 
two ophthalmologists. The examiners had undergone extensive interobserver reliability testing and maintained interobserver agreement at $95 \%$ for the early vascular changes of ROP and $99 \%$ for stage 1 ICROP or worse disease. ${ }^{8}$ In addition, for quality control, a retinal consultant examined $10 \%$ of study infants to corroborate findings.

\section{STATISTICAL METHODS}

Onset of ROP was defined as the time when ROP was first observed clinically. Unless otherwise stated onset of ROP was defined as onset of stage 1 ICROP. When onset is defined as the earliest vascular abnormalities defined in the SchafferQuinn classification it is clearly stated. The analysis was performed for right and left eye of study infants.

Analysis was undertaken by considering the following variables: BW, GA at birth (as determined on enrolment by physical assessment and maternal history), and age at onset of ROP. Unpaired $t$ tests, two tailed, were performed with significance defined as $\mathrm{p}=0.05$ or less.

\section{Results}

Nine hundred and fourteen infants were enrolled and complete acute phase ROP information was collected on 755 children ( $385 \mathrm{P}$ and $370 \mathrm{E}$ ). Of these 424 had BW $\leq 1500 \mathrm{~g}$ (216 P, $208 \mathrm{E})$. Among $P$ treated infants, 111 developed Stage 1 ICROP or worse in one or both eyes $(110 \mathrm{OD}$, 108 OS). For E treated infants, ROP developed in one or both eyes of 96 infants ( $94 \mathrm{OD}, 93 \mathrm{OS}$ ). ROP was therefore unilateral in nine infants (4 $P, 5 E$ ), occurring only in the right eye five times and only in the left eye four times. Right eye data are the subject of this report, however, results of the analysis are not different and no significant

Figure 1 The posinatal (weeks) at onset of ROP compared with the $G A$ at infants $(\nabla)$ and $96 E$ treated infants (O) who developed infants (O) who developed ROP. The best fit regression line for $P$ treated infants is represented by the solid line (slope -0.47) and for the $E$ treated group by a broken line (slope -0.48).

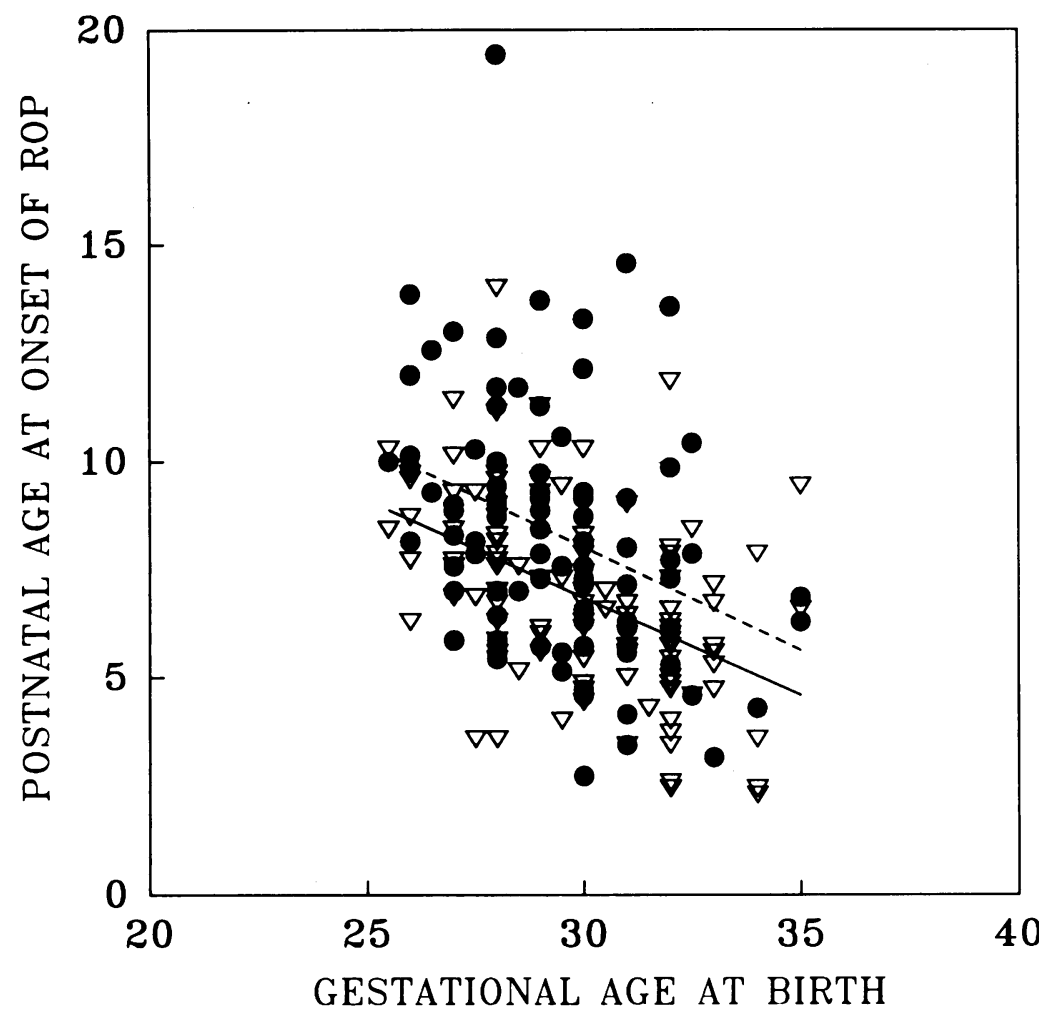

changes are noted if the analysis is done with the left eye data point.

Previous reports of this trial ${ }^{12}$ employed the Schaffer-Quinn classification and included the $13 \mathrm{P}$ treated and three $\mathrm{E}$ treated infants who developed ROP not severe enough to be characterised as ROP in the ICROP classification. These infants are not included in the analysis when ICROP is used.

Table 1 shows the similar distribution between $P$ and $E$ treated infants for $B W$ and GA as randomised ${ }^{3}$ and between those groups of $P$ and $E$ treated infants who developed ROP. The differences noted between $P$ and $E$ treated infants are not significant. For the entire study population there were no significant differences between inborn vs outborn status, 1 and 5 minute Apgar scores, or any of the recognised risk factors for ROP such as respiratory distress syndrome, bronchopulmonary dysplasia, patent ductus arteriosus, or intraventricular haemorrhage. The decrease in incidence of ROP associated with $\mathrm{E}$ treatment as well as the increased incidence of sepsis and necrotising enterocolitis in infants with BW of less than $1500 \mathrm{~g}$ who received prophylactic pharmacological $\mathrm{E}$ treatment for more than 7 days has been previously reported.'

Figure 1 presents, on the y axis, the postnatal age (weeks) at first observation of ROP in the right eye of $111 \mathrm{P}$ treated and $96 \mathrm{E}$ treated infants compared with their GA at birth. The best fit regression lines for the two populations are also shown ( $P$ treated infants, $r=0.47$ with a slope of $-0.47, \mathrm{p}<0.001 ; \mathrm{E}$ treated infants, $\mathrm{r}=0.35$, slope $=-0.48, p<0.001)$. The figure shows a consistent time lag for postnatal age at onset of ROP in infants treated with vitamin $E$. The mean age at onset of ROP in the right eye of $P$ treated infants with retinopathy is 6.9 weeks (SE $0 \cdot 2$ ) compared with a mean age of $8 \cdot 3$ weeks (SE $0.2)$ for $E$ treated infants with a mean E-P difference of 1.3 weeks (SE 0.3$) \quad(t=4.004$, $\mathrm{p}<0.0001)$. It is apparent from the figure that, regardless of study medication, infants born earlier in gestation develop ROP at a later postnatal age than infants born later in gestation (note negative slope of regression lines).

Also apparent in Figure 1 but not as easily discernible is the fact that infants born earlier in gestation develop ROP earlier after conception than infants born later in gestation. For example a child born at 27 weeks GA will develop ROP around 34-35 weeks postconceptional age (GA at birth + postnatal age) compared with a child born at 32 weeks who will develop ROP at 36-37 weeks postconceptional age. This is clearly seen in Figures 2 and 3 which plot the postnatal age at onset of ROP vs postconceptional age at onset of ROP for each $P$ treated infant (Fig 2) and for each $\mathrm{E}$ treated infant (Fig 3 ). The mean postconceptional age at onset of ROP in P treated infants is 36.7 weeks (SE 0.22 ) and in $E$ treated infants is 37.7 weeks $($ SE 0.30$)(t=2.68, p<0.01)$. The positive slope of the regression lines for both the $P$ (intercept $33 \cdot 1$, slope $+0 \cdot 52$ ) and $E$ treated (intercept $31 \cdot 5$, slope +0.75 ) populations again show that infants who develop ROP earlier after conception (y axis) also manifest the retinopathy sooner after birth ( $x$ axis). 


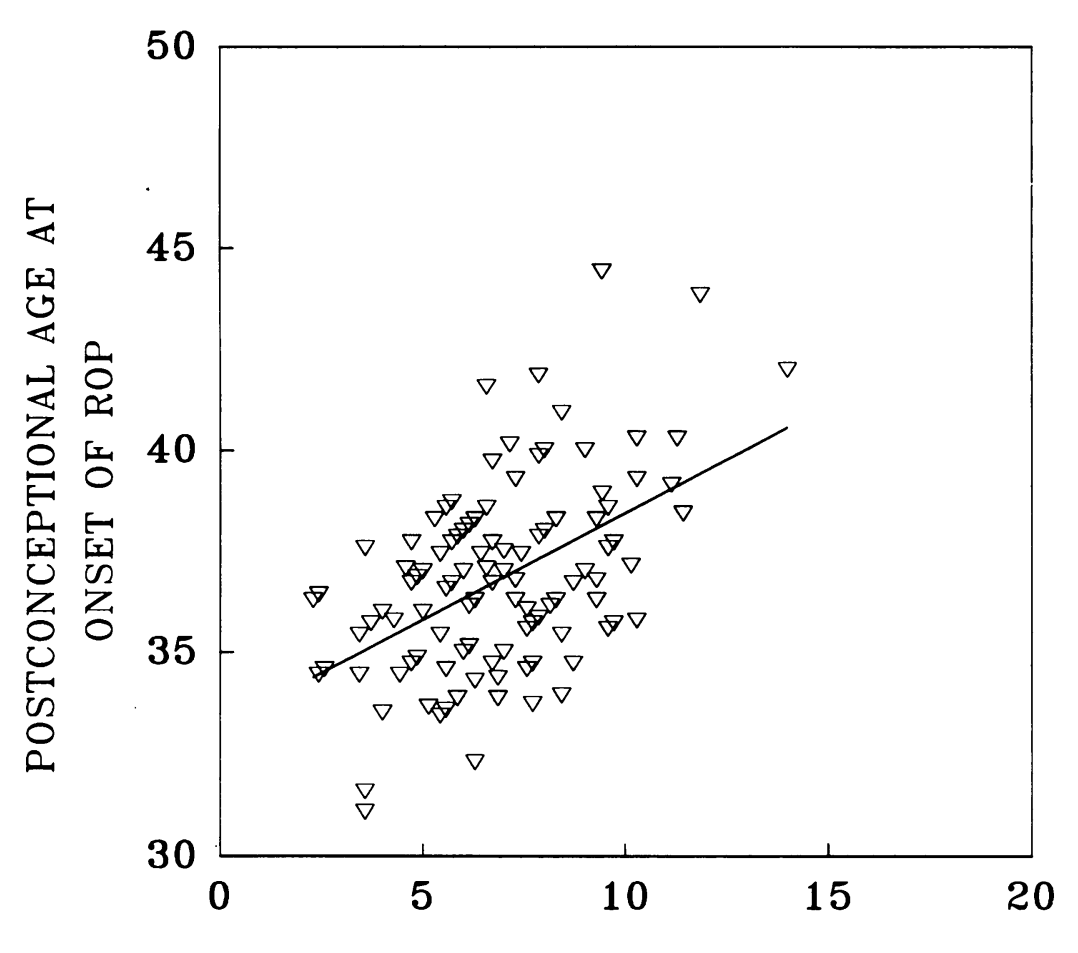

Figure 2 Comparison of postnatal age (weeks) at onset of ROP with

postconceptional age at onset postconceptional age at onset
of ROP for infants assigned to receive Placebo study medication. The best fit regression line for the $P$ treated infants is represented by the solid line (slope +0.52).

Figure 3 Comparison of postnatal age (weeks) at onset of ROP with postconceptional age at onset of ROP for infants assigned to receive vitamin $E$ study medication. The best fit regression line for the regression line for the
$E$ treated infants is $E$ treated infants is
represented by the broken line (slope +0.75).

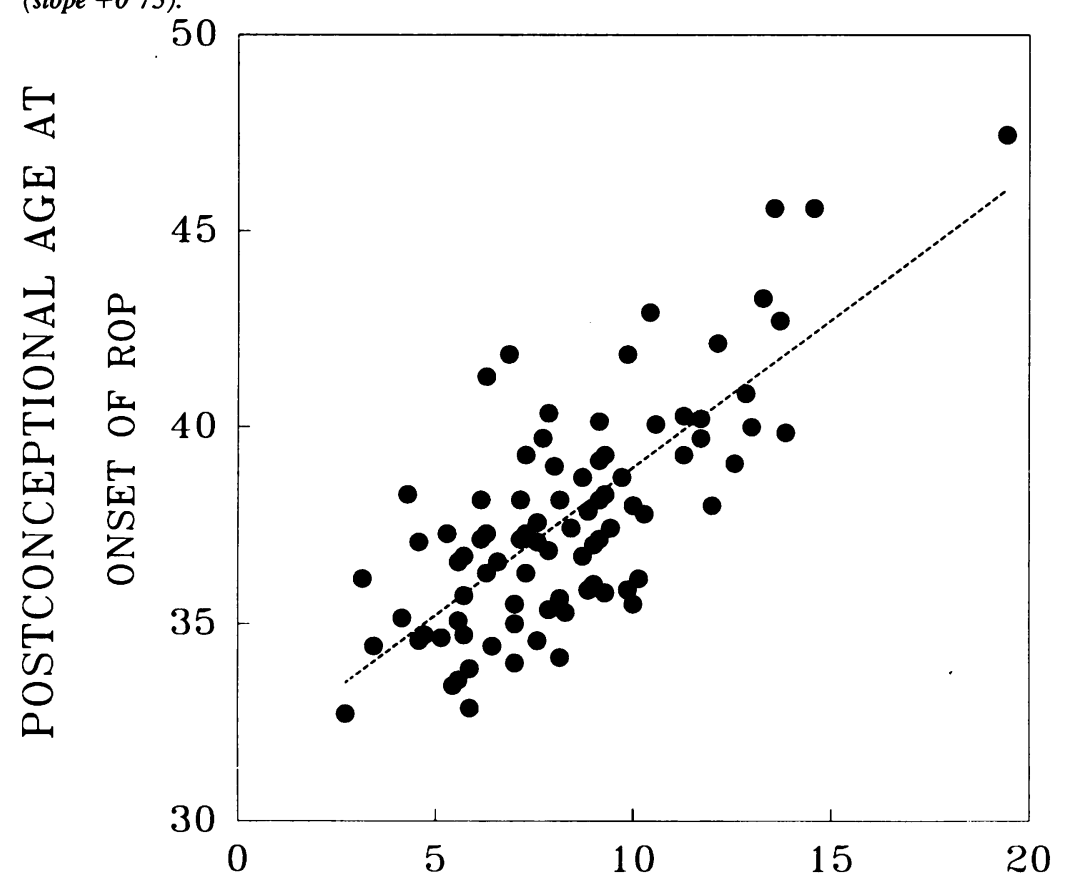

POSTNATAL AGE AT ONSET OF ROP examinations. Palmer, ${ }^{9}$ in a series of 175 infants, concluded that the optimal age for screening for ROP was between 7 and 9 weeks after birth. Flynn, ${ }^{10}$ in a larger series of 639 infants, found that most ROP was observed 'between 32 and 44 weeks postconceptional age.' He suggested using the term, 'conceptual age', to 'relate all infants to the same time axis' after conception. "In this report, we have concentrated on the complex relationship between 'conceptual age' and postnatal age when ROP is first observed. Our data are unique in their inclusion of infants with a wide range of BW and GA who were examined in the first week after birth, underwent weekly examinations, and who developed ROP. Such a population is no longer available for study because the incidence of ROP in the birth weight groups above $1250 \mathrm{~g}$ has fallen dramatically $^{12}$ as a result of ongoing improvements in both nutrition and perinatal/neonatal care. Without the inclusion of these more mature infants the accelerating effect on postnatal age at appearance of ROP exerted by perinatal insults early in gestation could not have been seen due to the homogeneity of very low birth weight populations (see Figs 2 and 3).

The earliest ophthalmoscopically visible signs of ROP are vascular abnormalities at the growing ends of the peripheral retinal vessels. ${ }^{1314}$ It is generally accepted that the insults that initiate this retinopathy occur during the perinatal period and are primarily related to degree of prematurity, exposure to an oxygen rich atmosphere, and hypoxic/ischaemic episodes during labour, delivery, and early extrauterine life. These events result in an increased production of oxidant radicals. ${ }^{15} 16$ Oxidant radical exposure appears to perturb the complex pattern of cellular events associated with retinal vascular maturation. These perturbations include alterations in membranes of vasoformative mesenchymal elements and nascent capillaries which lead to a delay in the normal time sequence of vascularisation ${ }^{1718}$ and which in some infants result in the early vascular abnormalities of ROP.

Vascularisation of the inner retinal layers, which are farthest from the choroidal circulation, develops in response to the increased oxygen needs of the differentiating retina. ${ }^{1314} 19$ Abnormalities of the developing vessels probably occur during periods of vessel growth and they appear to become manifest later after birth but earlier after conception in infants who are born very early compared with less early in gestation (see Fig 1). This suggests that both the state of retinal development and the time of exposure to the oxygen rich extrauterine environment, with its associated unphysiological perinatal events, influence the time at which the visible changes of ROP occur. If perinatal conditions ${ }^{1}$ (an oxygen rich environment, hyperoxia, hypoxia, hypothermia, hypercarbia), independent of ocular growth and development, were sufficient to initiate retinopathy one would expect to see ROP at a constant postnatal age regardless of GA at birth. In other words the regression line for Figure 1 relating GA at birth and postnatal age at onset of ROP would be parallel to the $x$ axis. This is not the case since postnatal age at onset of ROP 
increases with decreasing GA at birth (Fig 1). If on the other hand the stage of development of the retina solely determined the time when ROP becomes visible one would expect the appearance of ROP at a constant postconceptional age again regardless of GA at birth. For example if all ROP first occurred at 35 weeks after conception the baby born at 25 weeks would develop ROP at the same postconceptional age as the baby born at 30 or 35 weeks of gestation - that is, the postnatal age at onset of ROP would be at age 10 weeks for the 25 week GA infant, 5 weeks for the 30 week GA infant, and at birth for the 35 week GA infant. As is apparent from Figure 1 this is not the case since postconceptional age (GA at birth + postnatal age) at onset of ROP increases with increasing GA at birth.

This observation argues for a complex relationship between immaturity and events which occur during the transition to extrauterine life. We speculate that the most likely explanation for the greater delay in time from birth to appearance of ROP in the more immature infant, compared with the less prematurely born, is the fact that, in terms of thickness and stage of differentiation, the more immature retina takes longer to reach the point at which cellular metabolic demands require ongoing vascularisation. Onset of ROP nevertheless occurs sooner after conception in the baby who is very prematurely born compared with the more mature infant who manifests ROP later after conception but sooner after birth. The perinatal and neonatal insults which lead to initiation and progression of disease are not sustained by the more mature baby as early in the course of vascular development.

The data for $P$ treated infants as presented in Figure 2 emphasise the importance of both perinatal insults and stage of retinal development at introduction to an oxygen rich atmosphere in determining the age at onset of ROP. If there were no or minimal influence of perinatal events then for each week increase in postnatal age at onset of ROP there would be a corresponding increase of 1 week for the postconceptional age at onset of ROP. The slope of the line defining this relationship would be 1.00 and the regression line would intersect the y axis at 45 degrees. This is clearly not the case for data from $P$ treated infants shown in Figure 2 in which the slope of the line is 0.52 .

It appears that the relationship between postnatal and postconceptional age at onset of ROP has been altered by prophylactic treatment with $\mathrm{E}$. As seen in Figure 3 the slope of the line showing the data for $E$ treated infants is 0.75 , rather than 0.52 . This is what one would expect if the contribution of perinatal insults had been decreased, but not eliminated, by antioxidant treatment.

That both stage of development and perinatal events are important in determining age at onset of ROP differs somewhat from the conclusion of Fielder $e t a l^{20}$ that the 'age at which ROP is first seen is controlled predominantly by stage of development rather than neonatal events'. It should be pointed out however that these authors also noted that infants with gestational ages less than 28 weeks at birth developed ROP 2 weeks earlier after conception than later born infants ( 33.7 vs 35.7 weeks, respectively).

New vessel formation in the normally developing retina in both the intrauterine and extrauterine environment is believed to be governed by a graded hypoxic stimulus originating from hypoxia-related angiogenic factors in tissues near the junction of vascularised and non-vascularised retina. If the abnormal vessel formation in the retina with ROP results from oxygen-induced capillary die-back and the associated increase in hypoxia-related angiogenic factors, as suggested by Pat $\mathrm{Z}^{13}$ and Ashton, ${ }^{1+}$ then the area of differentiated retina requiring new growth (or regrowth) of vessels would be the limited area near the junction of vascularised and avascular retina. This band of relative hypoxia would be smallest in the least mature retinas (vascularised only into zone 1) and largest in those retinas with vessels extending well into zone 2 which occupies a much larger area. ROP should occur therefore sooner after conception in those infants born with retinal vascularisation into zone 2. One would not expect the postconceptional age at onset of ROP to be less in the infants born most prematurely with vascularisation confined to zone 1 , as was the case in our data (Figs 2 and 3). If however as in the spindle cell theory of ROP, ${ }^{18 \geq 1}$ the extent of insult to the avascular spindle cell-populated retina (which varies inversely with postconceptional age) determines the magnitude of angiogenic stimulus, then one would expect the magnitude of this stimulus to be greatest in the most prematurely born infants. Such infants would probably manifest more severe retinopathy sooner after conception but later after birth than their later born counterparts. Arguing from these premises our data are better explained by the spindle cell than by the capillary die-back theory of ROP.

Finally what is the significance of the time delay in ROP seen in E treated infants and what is its relevance for the clinician caring for the child at risk for ROP? As seen by the essentially parallel regression lines for $P$ and $E$ treated infants shown in Figure 1 the delay in onset of ROP caused by prophylactic antioxidant administration is remarkably consistent across the study population even for infants below 28 week GA. Kretzer and Hittner ${ }^{18}{ }^{21}$ have suggested that vitamin $\mathrm{E}$ would be minimally effective in preventing retinopathy in infants below 28 weeks of gestation because the developing interstitial retinol binding protein transport system cannot efficiently transport vitamin $\mathrm{E}$ from the outer to the inner retina prior to that point. However even in these smallest infants they found that vitamin E decreased the overall severity of disease and postponed the age at which sight threatening ROP was seen. The clinical data from this trial, as well as our earlier work, ${ }^{22}$ are consistent with this observation and suggest that when postnatal vitamin E does not prevent ROP it nevertheless delays its onset. This amounts to a considerable clinical advantage since the earlier the onset of ROP the more likely it is to progress to severe retinopathy. ${ }^{23}$ Furthermore the E related delay in time to peak disease which we observed in this clinical trial ${ }^{2+}$ 
would result in an infant who is older and probably more stable when cryotherapy for threshold $\mathrm{ROP}^{25}$ is indicated. As pointed out in our earlier report ${ }^{1}$ the optimal serum level and regimen of prophylactic $E$ supplementation is not certain, but should probably be one that achieves serum total $E$ levels in the physiological range $(1-3 \mathrm{mg} / \mathrm{dl})$ soon after birth. Serum E levels in the pharmacological range should not be used for prophylaxis but show promise for treatment of severe ROP. The exact mechanism of the delay observed in this study population is open for discussion but it probably hinges on the ability of vitamin E, a biological antioxidant, to stabilise membranes, decrease oxidant damage, and promote healing. ${ }^{12627}$ The authors wish to thank Michael E Breton, PhD and Dan
Montzka, MD for their help in statistical analysis, Mary Grous for Montzka, MD for their help in statistical analysis, Mary Grous for her research assistance, Glen Gole, MD for helpful suggestions
with manuscript, and David B Schaffer, MD, who performed a with manuscript, and David B Schaffer, MD, number of the eye examinations reported here.
This work was supported in part by the Retinal and Choroidal Disease Branch of the National Eye Institute, Grant no RO1EY01723. It was presented in part at the Association for Research in Vision and Ophthalmology Annual Meeting, May
1989, Sarasota, FL, USA.

1 Johnson L, Quinn GE, Abbasi S, et al. Effect of sustained pharmacologic vitamin $E$ levels on incidence and severity of retinopathy of prematurity: a controlled clinical trial $\mathcal{f}$ Pediatr 1989; 114: 827-38.

2 Johnson L, Bowen FW, Abbasi A, et al. Relationship of prolonged pharmacologic serum levels of vitamin E to prolonged pharmacologic serum levels of vitamin E to incidence of sepsis and necrotising enterocolitis in infants
with birth weight 1500 grams or less. Pediatrics 1985; 75: with birth

3 Schaffer DB, Johnson L, Quinn G, Boggs TR. A classification of retrolental fibroplasia to evaluate vitamin $\mathrm{E}$ therapy. of retrolental fibroplasia to eval
Ophthalmology 1979; 86: 1749-60.

4 Quinn GE, Schaffer DB, Johnson L. A revised classification of retinopathy of prematurity. Am $\mathcal{F}$ Ophthalmol 1982; 94: 744 9.

5 International Committee for the Classification of Retinopathy of Prematurity. An international classification of retinopathy of Prematurity. An international classification of retin
of prematurity. Arch Ophthalmol 1984; 102: 1130-4.

$6 \mathrm{Patz} \mathrm{A}$. The role of oxygen in retrolental fibroplasia. Graefes Arch Klin Exp Ophthalmol 1975; 195: 77-85.

7 McCormick A. The retinopathy of prematurity in the newborn. Curr Prob Pediatr 1977; 7:1-28.

8 Schaffer DB, Johnson L, Quinn GE, Abbasi S, Otis C, Bowen
FW. Vitamin E and retinopathy of prematurity: the ophthalmologist's perspective. March of Dimes Birth Defects: Original Article Series 1988; 24: 219-35.

9 Palmer EA. Optimal timing of examination for acute retrolental fibroplasia. Ophthalmology 1981; 88: 662-8.

10 Flynn JT. Acute proliferative retrolental fibroplasia: multivariate risk analysis. Trans Am Ophthalmol Soc 1983 81: 549-91.

11 Flynn JT. An international classification of retinopathy of prematurity: clinical experience. Ophthalmology 1985; 92 : 988-94.

12 Johnson LH, Quinn GE, Abbasi S, Bowen FW. Retinopathy of prematurity: prevalence and treatment over a 20 yea period at Pennsylvania Hospital. Doc Ophthalmol 1990; 74 213-22.

13 Patz A. Oxygen studies in retrolental fibroplasia. IV. Clinical and experimental observations. Am $\mathcal{F}$ Ophthalmol 1954; 38:

14 Ashton NB, Ward B, Serpell G. Effect of oxygen on developing retinal vessels with particular reference to the problem of retrolental fibroplasia. Brf Ophthalmol 1954; 38: 397-432.

15 McCord JM. Oxygen-derived free radicals in postischemic tissue injury. NEng f Med 1985; 312: 159-63.

16 Freeman BA, Crapo JD. Biology of disease: free radicals and tissue injury. Lab Invest 1982; 47:412-26.

17 Koutz CA Penn JS, Yip KF. Development of retinal vasculature in normoxic and hyperoxic rats. Invest Ophthat mol 1990; 31: 132.

18 Kretzer FL, Mehta RS, Johnson AT, Hunter DG, Brown ES Hittner HM. Vitamin E protects against retinopathy of prematurity through action on spindle cells. Nature 1984 ; 109: 793-5.

19 Foos RY, Kopelow SM. Development of retinal vasculature in paranatal infants. Surv Ophthalmol 1973; 18: 117-27. 20 Fielder AR, $\mathrm{Ng}$ YK, Levene MI. Retinopathy of prematurity:

21 age at onset. Arch Dis Child 1986; 61: 774-8. prematurity: interpretation and predictions. March of Dimes Original Article Series 1988; 24: 147-68.

22 Johnson L, Schaffer D, Boggs TR. The premature infant, vitamin E deficiency and retrolental fibroplasia. Am 7 Clin Nutr 1974; 27: 1158-73.

23 Schaffer DB, Metz HS, Palmer EA, Plotsky DF, Tung B. Prognostic factors in the natural course of retinopathy of prognostic factors in the natural course of

24 Quinn GE, Johnson L, Otis C, Schaffer DB, Bowen FW. Incidence, severity and time course of ROP in a randomized Incidence, severity and time course of ROP in a randomized
trial of vitamin E prophylaxis. Doc Ophthalmol 1990; 74: trial of

25 Cryotherapy for Retinopathy of Prematurity Cooperative Group. Multicenter trial of cryotherapy for retinopathy of prematurity: preliminary results. Arch Ophthalmol 1988; 106: 471-9.

26 Dormandy TL. Free-radical oxidation and antioxidants. Lancet 1978; i: 647-50.

27 Lucy JA, Functional and structural aspects of biological Lucy $\mathrm{JA}$, Functional and structural aspects of biological
membranes: a suggested structural role for vitamin $\mathrm{E}$ in the membranes: a suggested structural role for vitamin $\mathrm{E}$ in the Acad Sci 1972; 203: 4-11. 